\title{
A GENERALIZATION OF $J$-QUASIPOLAR RINGS
}

\author{
T. P. CALCI, S. HALICIOGLU, AND A. HARMANCI
}

Received 22 January, 2015

\begin{abstract}
In this paper we introduce a class of quasipolar rings which is a generalization of $J$ quasipolar rings. Let $R$ be a ring with identity. An element $a \in R$ is called $\delta$-quasipolar if there exists $p^{2}=p \in \operatorname{comm}^{2}(a)$ such that $a+p$ is contained in $\delta(R)$, and the ring $R$ is called $\delta$-quasipolar if every element of $R$ is $\delta$-quasipolar. We use $\delta$-quasipolar rings to extend some results of $J$-quasipolar rings. Then some of the main results of $J$-quasipolar rings are special cases of our results for this general setting. We give many characterizations and investigate general properties of $\delta$-quasipolar rings.
\end{abstract}

2010 Mathematics Subject Classification: 16S50; 16S70; 16 U99

Keywords: quasipolar ring, $\delta$-quasipolar ring, $\delta$-clean ring, $J$-quasipolar ring

\section{INTRODUCTION}

Throughout this paper all rings are associative with identity unless otherwise stated. Let $R$ be a ring. According to Koliha and Patricio [10], the commutant and double commutant of an element $a \in R$ are defined by $\operatorname{comm}(a)=\{x \in R \mid x a=a x\}$, $\operatorname{comm}^{2}(a)=\{x \in R \mid x y=y x$ for all $y \in \operatorname{comm}(a)\}$, respectively. If $R^{\text {qnil }}=$ $\{a \in R \mid 1+a x \in U(R)$ for every $x \in \operatorname{comm}(a)\}$ and $a \in R^{q n i l}$, then $a$ is said to be quasinilpotent (see [9]). The element $a$ is called quasipolar if there exists $p^{2}=p \in R$ such that $p \in \operatorname{comm}^{2}(a), a+p$ is invertible in $R$ and $a p \in R^{q n i l}$. Any idempotent $p$ satisfying the above conditions is called a spectral idempotent of $a$, and this term is borrowed from spectral theory in Banach algebra and it is unique for $a$.

Quasipolar rings have been studied by many ring theorists (see [1, 2, 5-7, 9, 10] and [15]). In [7], the element $a \in R$ is called nil-quasipolar if there exists $p^{2}=$ $p \in \operatorname{comm}^{2}(a)$ such that $a+p$ is nilpotent, the idempotent $p$ is called a nil-spectral idempotent of $a$. The ring $R$ is said to be nil-quasipolar if every element of $R$ is nilquasipolar. Recently, $J$-quasipolar rings are studied in [4]. The element $a$ is called $J$-quasipolar if there exists $p^{2}=p \in R$ such that $p \in \operatorname{comm}^{2}(a)$ and $a+p \in J(R)$, $p$ is called a $J$-spectral idempotent of $a$. The ring $R$ is said to be $J$-quasipolar if

The first author thanks the Scientific and Technological Research Council of Turkey (TUBITAK) for the financial support. 
every element of $R$ is $J$-quasipolar. Motivated by these, we introduce a new class of quasipolar rings which is a generalization of $J$-quasipolar rings. By using $\delta$ quasipolar rings, we extend some results of $J$-quasipolar rings.

An outline of the paper is as follows: Section 2 deals with $\delta$-quasipolar rings. We prove various basic characterizations and properties of $\delta$-quasipolar rings. It is proven that every $J$-quasipolar ring is $\delta$-quasipolar. We supply an example to show that all $\delta$-quasipolar rings need not be $J$-quasipolar. Among others the $\delta$-quasipolarity of Dorroh extensions and some classes of matrix rings are investigated. In Section 3, we introduce an upper class of $\delta$-quasipolar rings, namely, weakly $\delta$-quasipolar rings. We show that every direct summand of a weakly $\delta$-quasipolar ring is weakly $\delta$-quasipolar and every direct product of weakly $\delta$-quasipolar rings is weakly $\delta$-quasipolar, and we give some properties of such rings.

In what follows, $\mathbb{Z}$ and $\mathbb{Q}$ denote the ring of integers and the ring of rational numbers and for a positive integer $n, \mathbb{Z}_{n}$ is the ring of integers modulo $n$. For a positive integer $n$, let $\operatorname{Mat}_{n}(R)$ denote the ring of all $n \times n$ matrices and $T_{n}(R)$ the ring of all $n \times n$ upper triangular matrices with entries in $R$. We write $J(R)$ and $\operatorname{nil}(R)$ for the Jacobson radical of $R$ and the set of nilpotent elements of $R$, respectively.

\section{2. $\delta$-QUASIPOLAR RINGS}

In this section we introduce the concept of $\delta$-quasipolar rings and investigate some properties of such rings. We show that every quasipolar ring need not be $\delta$-quasipolar (Example 2). It is proven that every $J$-quasipolar ring is $\delta$-quasipolar and the converse does not hold in general (see Example 3). Among others we extend some results of $J$-quasipolar rings for this general setting.

A right ideal $I$ of the ring $R$ is said to be $\delta$-small in $R$ if whenever $R=I+K$ with $R / K$ singular right $R$-module for any right ideal $K$ then $R=K$. In [16], the ideal $\delta(R)$ is introduced as a sum of $\delta$-small right ideals of $R$. We begin with the equivalent conditions for $\delta(R)$ which is proved in [16, Theorem 1.6] for an easy reference for the reader.

Lemma 1. Given a ring $R$, each of the following sets is equal to $\delta(R)$.

(1) $R_{1}=$ the intersection of all essential maximal right ideals of $R$.

(2) $R_{2}=$ the unique largest $\delta$-small right ideal of $R$.

(3) $R_{3}=\left\{x \in R \mid x R+K_{R}=R\right.$ implies $K_{R}$ is a direct summand of $\left.R_{R}\right\}$.

(4) $R_{4}=\bigcap\{$ ideals $P$ of $R \mid R / P$ has a faithful singular simple module $\}$.

(5) $R_{5}=\{x \in R \mid$ for all $y \in R$ there exists a semisimple right ideal $Y$ of $R$ such that $\left.(1+x y) R \oplus Y=R_{R}\right\}$.

Now we give our main definition. 
Definition 1. Let $R$ be a ring. An element $a \in R$ is called $\delta$-quasipolar if there exists $p^{2}=p \in \operatorname{comm}^{2}(a)$ such that $a+p \in \delta(R)$ and $p$ is called a $\delta$-spectral idempotent. The ring $R$ is called $\delta$-quasipolar if every element of $R$ is $\delta$-quasipolar.

The following are examples for $\delta$-quasipolar rings.

Example 1. (1) Every semisimple ring and every Boolean ring is $\delta$-quasipolar. (2) Since $\delta(\mathbb{Q})=\mathbb{Q}, \mathbb{Q}$ is $\delta$-quasipolar. On the other hand, $\mathbb{Z}$ is not $\delta$-quasipolar since $\delta(\mathbb{Z})=0$.

One may suspects that every quasipolar ring is $\delta$-quasipolar. But the following example erases the possibility.

Example 2. Let $p$ be a prime integer with $p \geq 3$ and $R=\mathbb{Z}_{(p)}$ the localization of $\mathbb{Z}$ at the ideal $(p)$. By [4, Example 2.8], $R$ is a quasipolar ring. Since $J(R)=\delta(R)$, it is not $\delta$-quasipolar.

Let $S_{r}$ denote the right socle of the ring $R$, that is, $S_{r}$ is the sum of minimal right ideals of $R$. We now prove that the class of $J$-quasipolar rings is a subclass of $\delta$-quasipolar rings.

Lemma 2. If $R$ is a $J$-quasipolar ring, then $R$ is $\delta$-quasipolar. The converse holds if $S_{r} \subseteq J(R)$.

Proof. The first assertion is clear since $J(R) \subseteq \delta(R)$. Assume that $R$ is $\delta$-quasipolar. If $S_{r} \subseteq J(R)$, then $J(R) / S_{r}=J\left(R / S_{r}\right)=\delta(R) / S_{r}$ by [16, Corollary 1.7] and we have $J(R)=\delta(R)$. Hence, $R$ is $J$-quasipolar.

The converse of Lemma 2 is not true in general as the following example shows.

Example 3. Let $F$ be a field and consider the ring $R=\left[\begin{array}{ll}F & F \\ F & F\end{array}\right]$. Then $R$ is a semisimple ring and $R=\delta(R)$ and $J(R)=0$. Hence $R$ is $\delta$-quasipolar and it is not $J$-quasipolar.

Lemma 3. Let $R$ be a ring. Then we have the following.

(1) If $a, u \in R$ and $u$ is invertible, then $a$ is $\delta$-quasipolar if and only if $u^{-1} a u$ is $\delta$-quasipolar.

(2) The element $a \in R$ is $\delta$-quasipolar if and only $-1-a$ is $\delta$-quasipolar.

(3) If $R$ is a $\delta$-quasipolar ring with $\delta(R)=J(R)$, then the spectral idempotent for any invertible element in $R$ is the identity of $R$.

Proof. (1) Assume that $a$ is $\delta$-quasipolar. Let $p^{2}=p \in \operatorname{comm}^{2}(a)$ such that $a+p \in \delta(R)$. Let $x \in \operatorname{comm}\left(u^{-1} a u\right)$. Then $\left(u x u^{-1}\right) a=a\left(u x u^{-1}\right)$. Since $p \in$ $\operatorname{comm}^{2}(a),\left(u x u^{-1}\right) p=p\left(u x u^{-1}\right)$. Hence $\left(u^{-1} p u\right)^{2}=u^{-1} p u \in \operatorname{comm}^{2}\left(u^{-1} a u\right)$. Since $\delta(R)$ is an ideal of $R, u^{-1}(a+p) u=u^{-1} a u+u^{-1} p u \in \delta(R)$. Thus $u^{-1} a u$ is $\delta$-quasipolar. Conversely, if $u^{-1} a u$ is $\delta$-quasipolar, then by the preceding proof 
$u\left(u^{-1} a u\right) u^{-1}=a$ is $\delta$-quasipolar.

(2) Assume that $a$ is $\delta$-quasipolar. Let $p^{2}=p \in \operatorname{comm}^{2}(a)$ such that $a+p=r \in$ $\delta(R)$. Then $-1-a+(1-p)=-r \in \delta(R)$. Then $1-p \in \operatorname{comm}^{2}(-1-a)$ and $1-p$ is the spectral idempotent of $-1-a$. Conversely, if $-1-a$ is $\delta$-quasipolar, then from what we have proved that $-1-(-1-a)=a$ is quasipolar.

(3) Assume that $\delta(R)=J(R)$. Then $\delta$-quasipolarity of $R$ implies $J$-quasipolarity of $R$. So its proof can be directly obtained from [4, Example 2.2].

In [4, Corollary 2.3], it is proved that if $R$ is a $J$-quasipolar ring, then $2 \in J(R)$. In this direction we prove the following.

Lemma 4. If $R$ is a $\delta$-quasipolar ring, then $2 \in \delta(R)$.

Proof. For the identity 1 , there exists $p^{2}=p \in R$ such that $1+p \in \delta(R)$. Multiplying the latter by $p$, we have $2 p \in \delta(R)$. So $2=2(1+p)-2 p \in \delta(R)$.

Lemma 4 can be used to determine whether given rings are $\delta$-quasipolar.

Example 4. (1) The ring $\mathbb{Z}_{3}$ is a semisimple ring and $\delta$-quasipolar but the ring $R=\left[\begin{array}{cc}\mathbb{Z}_{3} & \mathbb{Z}_{3} \\ 0 & \mathbb{Z}_{3}\end{array}\right]$ is not $\delta$-quasipolar since $\delta(R)=\left[\begin{array}{ll}0 & \mathbb{Z}_{3} \\ 0 & \mathbb{Z}_{3}\end{array}\right]$ and 2 does not contained in $\delta(R)$.

(2) Let $R=\left\{\left(a_{i j}\right) \in \mathrm{T}_{n}\left(\mathbb{Z}_{3}\right) \mid a_{11}=a_{22}=\cdots=a_{n n}\right\}$. $\mathbb{Z}_{3}$ is $\delta$-quasipolar but $R$ is not since $\delta(R)=\left\{\left(a_{i j}\right) \in \mathrm{T}_{n}\left(\mathbb{Z}_{3}\right) \mid a_{11}=a_{22}=\cdots=a_{n n}=0\right\}$ and 2 does not contained in $\delta(R)$.

Recall that a ring $R$ is called local if it has only one maximal left ideal, equivalently, maximal right ideal.

Proposition 1. Let $R$ be a local ring. If $R / J(R) \cong \mathbb{Z}_{2}$, then $R$ is $\delta$-quasipolar.

Proof. Let $a \in R$. If $a \in J(R)$, it is clear. Assume that $a \notin J(R)$. Since $R$ is local, $a$ is invertible. Hence $a+1 \in \delta(R)$ by $\delta(R)=J(R)$.

A ring $R$ is said to be clean [12] if for each $a \in R$ there exists $e^{2}=e \in R$ such that $a-e$ is invertible, and $R$ is called strongly clean [13] provided that every element of $R$ can be written as the sum of an idempotent and an invertible element that commute.

Example 5. Let $R=\left\{\left(q_{1}, q_{2}, q_{3}, \ldots, q_{n}, a, a, a, \ldots\right) \mid n \geq 1 ; q_{i} \in \mathbb{Q} ; a \in \mathbb{Z}_{(2)}\right\}$. Then $R$ is strongly clean but not quasipolar (see [15, Example 3.4(3)]). Therefore $R$ is not $J$-quasipolar since every $J$-quasipolar ring is quasipolar. On the other hand, since $S_{r}=0$ and $\delta(R) / S_{r}=J(R) / S_{r}, \delta(R)=J(R)$. Thus $R$ is not $\delta$-quasipolar.

In [4, Theorem 2.9], it is shown that if the ring $R$ is $J$-quasipolar, then $R / J(R)$ is Boolean and idempotents in $R / J(R)$ lift $R$. We have the following result for $\delta$ quasipolar rings. 
Theorem 1. If $R$ is a $\delta$-quasipolar ring, then $R / \delta(R)$ is a Boolean ring and idempotents in $R / \delta(R)$ lift $R$.

Proof. Let $\bar{a} \in R / \delta(R)$. There exists $p^{2}=p \in \operatorname{comm}^{2}(-1+a)$ such that $-1+$ $a+p \in \delta(R)$. Hence $\bar{a}=\overline{1-p}$ is an idempotent in $R / \delta(R)$ and $R / \delta(R)$ is a Boolean ring. Let $\bar{a}^{2}=\bar{a} \in R / \delta(R)$. Then there exists $p^{2}=p \in \operatorname{comm}^{2}(-a)$ such that $-a+p \in \delta(R)$. This yields $\bar{a}=\bar{p}$, as asserted.

The concept of $\delta_{r}$-clean rings are defined in [8]. A ring $R$ is called $\delta_{r}$-clean if for every element $a \in R$ there exists an idempotent $e \in R$ such that $a-e \in \delta(R)$. A ring is abelian if all idempotents are central.

Lemma 5. If $R$ is a $\delta$-quasipolar ring, then it is $\delta_{r}$-clean. The converse holds if $R$ is abelian.

Proof. Let $R$ be a $\delta$-quasipolar ring and $a \in R$. There exists $p^{2}=p \in \operatorname{comm}^{2}$ $(-1+a)$ such that $-1+a+p \in \delta(R)$. Then $a-(1-p) \in \delta(R)$. For the converse, assume that $R$ is abelian. Let $a \in R$. There exists an idempotent $e$ such that $1+a-$ $e \in \delta(R)$. By assumption, $1-e$ is a central idempotent and so $1-e \in \operatorname{comm}^{2}(a)$.

Recall that a ring $R$ is exchange if for every $a \in R$, there exists an idempotent $e \in a R$ such that $1-e \in(1-a) R$. Namely, von Neumann regular rings and clean rings are exchange.

Corollary 1. Let $R$ be a $\delta$-quasipolar ring. Then

(1) $R$ is an exchange ring.

(2) $R / \delta(R)$ is a clean ring.

Proof. (1) Let $R$ be a $\delta$-quasipolar ring. By Lemma 5, $R$ is a $\delta_{r}$-clean ring. By [8, Theorem 2.2(2)], every $\delta_{r}$-clean ring is an exchange ring.

(2) By Theorem $1, R / \delta(R)$ is Boolean, therefore, it is clean.

Corollary 2. Consider following conditions for a ring $R$.

(1) $R$ is $\delta$-quasipolar and $\delta(R)=0$.

(2) $R$ is Boolean.

(3) $R$ is von Neumann regular and $\delta$-quasipolar.

Then $(1) \Rightarrow(2) \Rightarrow(3)$.

Proof. (1) $\Rightarrow$ (2) Assume that $R$ is $\delta$-quasipolar and $\delta(R)=0$. By Theorem $1, R$ is Boolean.

(2) $\Rightarrow$ (3) Assume that $R$ is Boolean. Then it is commutative with characteristic 2 and $a^{2}+a=0 \in \delta(R)$ and $a^{2}=a=a^{3}$ for all $a \in R$. Hence $R$ is von Neumann regular and $\delta$-quasipolar.

Strongly $J$-clean rings were introduced by Chen in [3]. For a ring $R$ the element $a \in R$ is called $J$-clean if $a$ is the sum of an idempotent and a radical element in 
its Jacobson radical. The ring $R$ is called $J$-clean if every element is a sum of an idempotent and a radical element.

Theorem 2. If $R$ is an abelian $J$-clean ring, then it is $\delta$-quasipolar.

Proof. Let $a \in R$. Then we have $-a \in R$. Since $R$ is $J$-clean, there exist $e^{2}=$ $e \in R$ and $j \in J(R)$ such that $-a=e+j$. Hence $a+e \in J(R)$. Since $R$ is abelian, $e^{2}=e \in \operatorname{comm}^{2}(a)$ and $J(R) \subseteq \delta(R), R$ is $\delta$-quasipolar as asserted.

All $\delta$-quasipolar rings need not be Boolean and the converse statement of Theorem 2 is not true in general.

Example 6. The ring $\mathbb{Z}_{3}$ is semisimple and so $\mathbb{Z}_{3}=\delta\left(\mathbb{Z}_{3}\right)$. Therefore $\mathbb{Z}_{3}$ is $\delta$ quasipolar, but it is neither Boolean nor $J$-clean.

In [4, Proposition 2.11], it is shown that a ring $R$ is local and $J$-quasipolar if and only if $R$ is $J$-quasipolar with only trivial idempotents if and only if $R / J(R) \cong \mathbb{Z}_{2}$. We have the following for $\delta$-quasipolar rings.

Proposition 2. Let $R$ be a ring with only trivial idempotents. Then $R$ is $\delta$ quasipolar if and only if $R / \delta(R) \cong \mathbb{Z}_{2}$.

Proof. Assume that $R$ is $\delta$-quasipolar. Let $a \in R$. There exists an idempotent $p \in \operatorname{comm}^{2}(a)$ such that $-a+p \in \delta(R)$. By hypothesis $p=1$ or $p=0$. If $\delta(R)=0$, then $R / \delta(R) \cong \mathbb{Z}_{2}$. Suppose that $\delta(R) \neq 0$. For any $a \in R \backslash \delta(R), \bar{a}=\overline{1} \in R / \delta(R)$. Hence $R / \delta(R) \cong \mathbb{Z}_{2}$. Conversely, suppose that $R / \delta(R)$ is isomorphic to $\mathbb{Z}_{2}$ by isomorphism $f$. Let $a \in R \backslash \delta(R)$. Then $f(-\bar{a})=\overline{1} \in \mathbb{Z}_{2}$. Then $f(-\bar{a})=f(\overline{1})$ implies $-\bar{a}-\overline{1} \in \operatorname{Ker} f=0$. Hence $-\bar{a}=\overline{1}$. That is, $a+1 \in \delta(R)$. Thus $R$ is $\delta$-quasipolar.

Recall that a ring $R$ is called strongly $\pi$-regular if for every element $a$ of $R$ there exist a positive integer $n$ (depending on $a$ ) and an element $x$ of $R$ such that $a^{n}=$ $a^{n+1} x$, equivalently, an element $y$ of $R$ such that $a^{n}=y a^{n+1}$. In spite of the fact that $J(R)$ is contained in both $\delta(R)$ and $R^{q n i l}$, no comparings between $\delta(R)$ and $R^{q n i l}$ exist. Strongly $\pi$-regular rings play crucial role in this direction.

Proposition 3. Let $R$ be a $\delta$-quasipolar ring and $\delta(R)=J(R)$. Then $R$ is strongly $\pi$-regular if and only if $J(R)=R^{q n i l}=\operatorname{nil}(R)=\delta(R)$.

Proof. Necessity. Let $a \in R^{q n i l}$. Then for any $x \in \operatorname{comm}(a), 1-a x$ is invertible. By hypothesis, there exist a positive integer $m$ and $b \in R$ such that $a^{m}=a^{m+1} b$. Since $b \in \operatorname{com} m(a)$ by [11, Page 347, Exercise 23.6(1)], $a^{m}=0$. Hence $a \in \operatorname{nil}(R)$ and so $R^{q n i l} \subseteq \operatorname{nil}(R)$. To prove $\operatorname{nil}(R) \subseteq \delta(R)$, let $a \in \operatorname{nil}(R)$. By hypothesis there exists $p^{2}=p \in \operatorname{comm}^{2}(1-a)$ such that $1-a+p \in \delta(R)$. Since $1-a$ is invertible, $p=1$ by Lemma 3 (3). Hence $2-a \in \delta(R)$. Also $2 \in \delta(R)$ by Lemma 4, we then have $a \in \delta(R)$. 
Sufficiency. Let $a \in R$. There exists $p^{2}=p \in \operatorname{comm}^{2}(-1+a)$ such that $-1+$ $a+p \in \delta(R)$. Set $u=-1+a+p \in \operatorname{nil}(R)$. Then $a+p$ is invertible and $a p=u p$ is nilpotent so that $a^{n} p=0$ for some positive integer $n$. So $a^{n}=a^{n}(1-p)=$ $(u+(1-p))^{n}(1-p)=(u+1)^{n}(1-p)=(a+p)^{n}(1-p)=(1-p)(a+p)^{n}$. By [13, Proposition 1], $a$ is strongly $\pi$-regular. This completes the proof.

Let $R$ and $V$ be rings and $V$ be an $(R, R)$-bimodule that is also a ring with $(v w) r=$ $v(w r),(v r) w=v(r w)$, and $(r v) w=r(v w)$ for all $v, w \in V$ and $r \in R$. The Dorroh extension $D(R, V)$ of $R$ by $V$ defined as the ring consisting of the additive abelian group $R \oplus V$ with multiplication $(r, v)(s, w)=(r s, r w+v s+v w)$ where $r, s \in R$ and $v, w \in V$.

Uniquely clean rings were introduced by Nicholson and Zhou in [14]. A ring $R$ is uniquely clean in case for any $a \in R$ there exists a unique idempotent $e \in R$ such that $a-e \in R$ is invertible. In [8], among others, uniquely $\delta_{r}$-clean rings are studied. A ring $R$ is called uniquely $\delta_{r}$-clean if for every element $a \in R$ there exists a unique idempotent $e \in R$ such that $a-e \in \delta(R)$. Uniquely clean Dorroh extensions in [14, Proposition 7] and uniquely $\delta_{r}$-clean Dorroh extensions in [8, Proposition 3.11] are considered. Now we consider $\delta$-quasipolar Dorroh extensions.

Proposition 4. Let $R$ be a ring. Then we have the following.

(1) If $D(R, V)$ is $\delta$-quasipolar, then $R$ is $\delta$-quasipolar.

(2) If the following conditions are satisfied, then $D(R, V)$ is $\delta$-quasipolar.

(i) $R$ is $\delta$-quasipolar;

(ii) $e^{2}=e \in R$, then $e v=$ ve for all $v \in V$;

(iii) $V=\delta(V)$.

Proof. (1) Let $r \in R$. There exists $e^{2}=e \in D(R, V)$ such that $e \in \operatorname{comm}^{2}(r, 0)$ and $(r, 0)+e \in \delta(D(R, V))$. Since $e \in D(R, V), e$ has the form such that $(p, v)^{2}=$ $(p, v)$ and $p^{2}=p$. Then $e=(p, v) \in \operatorname{comm}^{2}(r, 0)$ implies that $p \in \operatorname{comm}^{2}(r)$ and $r+p \in \delta(R)$ since $(r+p, v) \in \delta(D(R, V))$ and by [8, Proposition 3.11]. Hence $R$ is $\delta$-quasipolar.

(2) Assume that (i), (ii) and (iii) hold. Let $(r, v) \in D(R, V)$. There exists $p^{2}=$ $p \in \operatorname{comm}^{2}(r)$ such that $r+p \in \delta(R)$. By (iii), $(0, V) \subseteq \delta(D(R, V))$. Then $(r, v)+$ $(p, 0)=(r+p, v) \in \delta(D(R, V))$. To see that $(p, 0) \in \operatorname{comm}^{2}((r, v))$, let $(a, b) \in$ $D(R, V)$ and $(a, b)(r, v)=(r, v)(a, b)$. Then $a r=r a$ and so $a p=p a$ since $p \in$ $\operatorname{comm}^{2}(r)$. Also $p b=b p$ by (ii). Therefore we have $(p, 0)(a, b)=(a, b)(p, 0)$ that is $(p, 0) \in \operatorname{comm}^{2}((r, v))$.

As an application of Dorroh extensions we consider the following example. This example also shows that in Proposition 4 (2), the conditions (i), (ii) and (iii) are not superfluous.

Example 7. Consider the ring $D(\mathbb{Z}, \mathbb{Q})$. Then $D(\mathbb{Z}, \mathbb{Q}) \cong \mathbb{Z} \times \mathbb{Q}$. Then $\delta(\mathbb{Z} \times$ $\mathbb{Q})=(0) \times \mathbb{Q}$. Since $\mathbb{Z}$ is not $\delta$-quasipolar, $D(\mathbb{Z}, \mathbb{Q})$ is not $\delta$-quasipolar. 
Let $R$ and $S$ be any ring and $M$ an $(R, S)$-bimodule. Consider the ring of the formal upper triangular matrix $\operatorname{ring} T=\left[\begin{array}{cc}R & M \\ 0 & S\end{array}\right]$. It is well known that $\delta(T) \subseteq$ $\left[\begin{array}{cc}\delta(R) & M \\ 0 & \delta(S)\end{array}\right]$. However, if $M=R=S=F$ is a field, then $\delta(T)=\left[\begin{array}{ll}0 & F \\ 0 & F\end{array}\right]$.

The following example illustrates the $\delta$-quasipolarity of full matrix rings and upper triangular matrix rings depend on the coefficient ring.

Example 8. (1) Consider the ring $R=\left[\begin{array}{cc}\mathbb{Z}_{2} & \mathbb{Z}_{2} \\ 0 & \mathbb{Z}_{2}\end{array}\right]$. Then $J(R)=\left[\begin{array}{cc}0 & \mathbb{Z}_{2} \\ 0 & 0\end{array}\right]$ and $\delta(R)=\left[\begin{array}{ll}0 & \mathbb{Z}_{2} \\ 0 & \mathbb{Z}_{2}\end{array}\right] . R$ is $\delta$-quasipolar.

(2) As noted in Example 4, the ring $\mathbb{Z}_{3}$ is semisimple and therefore $\delta$-quasipolar. However, the ring $\left[\begin{array}{cc}\mathbb{Z}_{3} & \mathbb{Z}_{3} \\ 0 & \mathbb{Z}_{3}\end{array}\right]$ is not $\delta$-quasipolar.

(3) Let $A=\left[\begin{array}{ll}0 & 1 \\ 0 & 0\end{array}\right] \in \operatorname{Mat}_{2}(\mathbb{Z})$. For any $P^{2}=P \in \operatorname{comm}^{2}(A)$, the matrix $P$ has the form $P=\left[\begin{array}{ll}x & y \\ 0 & x\end{array}\right]$ with $x^{2}=x$ and $2 x y=y$ where $x, y \in \mathbb{Z}$. This would imply that $P$ is the zero matrix or the identity matrix. Since $\delta(\mathbb{Z})=0$, $\delta\left(\operatorname{Mat}_{2}(\mathbb{Z})\right)=0$. In consequence, $A+P$ can not be in $\delta\left(\operatorname{Mat}_{2}(\mathbb{Z})\right)$. Therefore $\operatorname{Mat}_{2}(\mathbb{Z})$ is not $\delta$-quasipolar.

(4) Let $A=\left[\begin{array}{ll}1 & 0 \\ 0 & 0\end{array}\right] \in T_{2}(\mathbb{Z})$. The idempotents of $T_{2}(\mathbb{Z})$ are zero, identity, $\left[\begin{array}{ll}1 & 0 \\ 0 & 0\end{array}\right],\left[\begin{array}{ll}0 & 0 \\ 0 & 1\end{array}\right],\left[\begin{array}{ll}0 & y \\ 0 & 1\end{array}\right]$ and $\left[\begin{array}{ll}1 & y \\ 0 & 0\end{array}\right]$ where $y$ is an arbitrary integer. Since $A$ commutes with only zero, identity, $\left[\begin{array}{ll}1 & 0 \\ 0 & 0\end{array}\right]$ and $\left[\begin{array}{ll}0 & 0 \\ 0 & 1\end{array}\right]$, among these idempotents there is no idempotent $P$ such that $A+P \in \delta\left(T_{2}(\mathbb{Z})\right)$ since $\delta\left(T_{2}(\mathbb{Z})\right)=\left[\begin{array}{ll}0 & \mathbb{Z} \\ 0 & 0\end{array}\right]$. Hence $T_{2}(\mathbb{Z})$ is not $\delta$-quasipolar.

\section{WEAKLY $\delta$-QUASIPOLAR RINGS}

In this section, we introduce an upper class of $\delta$-quasipolar rings, namely, weakly $\delta$-quasipolar rings, and we give some properties of such rings.

Definition 2. Let $R$ be a ring and $a \in R$. The element $a$ is called weakly $\delta$ quasipolar if there exists $p^{2}=p \in \operatorname{comm}(a)$ such that $a+p \in \delta(R)$, and $p$ is called a weakly $\delta$-spectral idempotent. A ring $R$ is called weakly $\delta$-quasipolar if every element of $R$ is weakly $\delta$-quasipolar. 
An element of a ring is called strongly $J$-clean [3] provided that it can be written as the sum of an idempotent and an element in its Jacobson radical that commute. A ring is strongly $J$-clean in case each of its elements is strongly $J$-clean.

Example 9. (1) Every semisimple ring and every Boolean ring is weakly $\delta$-quasipolar, since $\delta$-quasipolar rings are weakly $\delta$-quasipolar.

(2) Every strongly $J$-clean ring is weakly $\delta$-quasipolar.

Proposition 5. Let $f: R \rightarrow S$ be a surjective ring homomorphism. If $R$ is weakly $\delta$-quasipolar, then $S$ is weakly $\delta$-quasipolar.

Proof. Let $s \in S$ with $s=f(r)$ where $r \in R$. There exists an idempotent $p \in$ $\operatorname{comm}(r)$ such that $r+p \in \delta(R)$. Let $q=f(p)$. Then $q^{2}=q \in \operatorname{comm}(f(r))=$ $\operatorname{comm}(s)$. By [16], $f(\delta(R)) \subseteq \delta(S)$. Then $s+q=f(r)+f(p)=f(r+p) \in$ $f(\delta(R)) \subseteq \delta(S)$. Hence $S$ is weakly $\delta$-quasipolar.

Corollary 3. Every direct summand of a weakly $\delta$-quasipolar ring is weakly $\delta$ quasipolar.

Proposition 6. Let $R=\prod_{i=1}^{n} R_{i}$ be a finite direct product of rings. $R$ is weakly $\delta$-quasipolar if and only if each $R_{i}$ is weakly $\delta$-quasipolar for $(i=1,2, \ldots, n)$.

Proof. One way is clear from Corollary 3. We may assume that $n=2$ and $R_{1}$ and $R_{2}$ are weakly $\delta$-quasipolar. Let $a=\left(x_{1}, x_{2}\right) \in R$. There exist idempotents $p_{i} \in \operatorname{comm}\left(x_{i}\right)$ such that $x_{i}+p_{i} \in \delta\left(R_{i}\right)$ for $(i=1,2)$. Then $p=\left(p_{1}, p_{2}\right)$ is an idempotent in $R$ and $p \in \operatorname{comm}(a)$ and $a+p \in \delta(R)$. Hence $R$ is weakly $\delta$ quasipolar.

In [8], Gurgun and Ozcan introduce and investigate properties of $\delta_{r}$-clean rings. Motivated by this work strongly $\delta_{r}$-clean rings can be defined as follows.

Definition 3. An element $x \in R$ is called strongly $\delta_{r}$-clean provided that there exist an idempotent $e \in R$ and an element $w \in \delta_{r}$ such that $x=e+w$ and $e w=w e$. A ring $R$ is called strongly $\delta_{r}$-clean in case every element in $R$ is strongly $\delta_{r}$-clean.

Any strongly $J$-clean ring is strongly $\delta_{r}$-clean. But the converse need not be true, for example any commutative semisimple ring which is not a Boolean ring is such a ring.

Note that in the following theorem it is proved that the notions of strongly $\delta_{r}$-clean rings and weakly $\delta$-quasipolar rings coincide.

Theorem 3. Let $R$ be a ring. Then $R$ is a weakly $\delta$-quasipolar ring if and only if it is strongly $\delta_{r}$-clean.

Proof. Let $R$ be a weakly $\delta$-quasipolar ring and $a \in R$. There exits $p^{2}=p \in$ $\operatorname{comm}(-1+a)$ such that $-1+a+p \in \delta(R)$. Then $a-(1-p) \in \delta(R)$ and $a(1-p)=$ $(1-p) a$. Hence $R$ is a strongly $\delta_{r}$-clean ring. Conversely, assume that $R$ is a strongly 
$\delta_{r}$-clean ring. Let $a \in R$. Since $-a \in R$, by assumption there exists an idempotent $p \in R$ such that $-a-p \in \delta(R)$ and $(-a) p=p(-a)$. So $R$ is a weakly $\delta$-quasipolar ring.

Theorem 3 states that the weakly $\delta$-quasipolarity of a ring is equivalent to the strongly $\delta_{r}$-cleanness of this ring. The following example reveals that a weakly $\delta$ quasipolar element is different from a strongly $\delta_{r}$-clean element.

Example 10. Let $R=\mathbb{Z}$ and $a=1 \in R$. There exists no idempotent $p$ such that $a+p \in \delta(R)$. Then $a$ is not weakly $\delta$-quasipolar. Let $p=1 \in R$. Since $a-p \in$ $\delta(R), a$ is strongly $\delta_{r}$-clean. On the other hand, if $a=-1 \in R$, then there exists no idempotent $p$ such that $a-p \in \delta(R)$. Then $a$ is not strongly $\delta_{r}$-clean. Let $p=1 \in R$. Since $a+p \in \delta(R), a$ is weakly $\delta$-quasipolar.

Theorem 4. Let $R$ be a local ring with non-zero maximal ideal. Then the following are equivalent.

(1) $R$ is weakly $\delta$-quasipolar;

(2) $R$ is strongly $J$-clean;

(3) $R$ is uniquely clean;

(4) $R / J(R) \cong \mathbb{Z}_{2}$;

(5) $R / \delta(R) \cong \mathbb{Z}_{2}$.

Proof. Let $R$ be a local ring with non-zero maximal ideal.

(1) $\Leftrightarrow$ (2) Assume that $R$ is weakly $\delta$-quasipolar. Let $a \in R$. There exists $p^{2}=p \in$ $\operatorname{comm}(-1+a)$ such that $-1+a+p \in \delta(R)$. Then $a-(1-p) \in \delta(R)$. Since $p \in$ comm $(-1+a), p a=a p$. Hence $R$ is strongly $J$-clean by $J(R)=\delta(R)$. Similarly, the rest is clear.

(2) $\Leftrightarrow$ (3) follows from [3, Lemma 4.2].

(3) $\Leftrightarrow$ (4) follows from [14, Theorem 15].

(1) $\Rightarrow(5)$ Let $R$ be weakly $\delta$-quasipolar and $\overline{0} \neq \bar{a}=a+\delta(R) \in R / \delta(R)$, we show that $\bar{a}=\overline{1}$. Then there exists an idempotent $p \in R$ such that $-a+p \in \delta(R)$ and $p^{2}=p \in \operatorname{comm}(-a)$. Since $R$ is a local, $p=0$ or $p=1$. If $p=0$, this contradicts $\overline{0} \neq \bar{a}$. Therefore $p=1$. It follows that $\bar{a}=\overline{1}$.

(5) $\Rightarrow$ (1) It follows from Proposition 2.

\section{ACKNOWLEDGEMENT}

The authors would like to thank the referee for his/her valuable suggestions which contributed to improve the presentation of this paper.

\section{REFERENCES}

[1] M. B. Calci, S. Halicioglu, and A. Harmanci, "A Class of J-Quasipolar Rings." J. Algebra Relat. Topics, vol. 3, no. 2, pp. 1-15, 2015.

[2] M. B. Calci, B. Ungor, and A. Harmanci, "Central Quasipolar Rings." Rev. Colombiana Mat, vol. 49, no. 2, pp. 281-292, 2015. 
[3] H. Chen, "On strongly J-clean rings." Comm. Algebra, vol. 38, no. 10, pp. 3790-3804, 2010, doi: 10.1080/00927870903286835.

[4] J. Cui and J. Chen, "A class of quasipolar rings.” Comm. Algebra, vol. 40, no. 12, pp. 4471-4482, 2012, doi: 10.1080/00927872.2011.610854.

[5] J. Cui and J. Chen, "Pseudopolar matrix rings over local rings." J. Algebra Appl., vol. 13, no. 3, pp. 1350 109, 12, 2014, doi: 10.1142/S0219498813501090.

[6] O. Gurgun, S. Halicioglu, and A. Harmanci, "Quasipolar Subrings of $3 \times 3$ Matrix Rings." An. St. Univ. Ovidius Constantia, vol. 21, no. 3, pp. 133-146, 2013, doi: 0.2478/auom-2013-0048.

[7] O. Gurgun, S. Halicioglu, and A. Harmanci, "Nil-quasipolar rings." Bol. Soc. Mat. Mex., vol. 20, no. 1, pp. 29-38, 2014, doi: 10.1007/s40590-014-0005-y.

[8] O. Gurgun and A. C. Ozcan, "A class of uniquely (strongly) clean rings." Turk. J. Math., vol. 38, pp. 40-51, 2014, doi: 10.3906/mat-1209-9.

[9] R. E. Harte, "On quasinilpotents in rings." Panamer. Math. J., vol. 1, pp. 10-16, 1991.

[10] J. J. Koliha and P. Patricio, "Elements of rings with equal spectral idempotents." J. Aust. Math. Soc., vol. 72, no. 1, pp. 137-152, 2002.

[11] T. Y. Lam, First course in noncommutative rings. New York: Springer, 2001.

[12] W. K. Nicholson, "Lifting idempotents and exchange rings." Trans. Amer. Math. Soc., vol. 229, pp. 269-278, 1977.

[13] W. K. Nicholson, "Strongly clean rings and fitting's lemma." Comm. Algebra, vol. 27, no. 8, pp. 3583-3592, 1999, doi: 10.1080/00927879908826649.

[14] W. K. Nicholson and Y. Zhou, "Rings in which elements are uniquely the sum of an idempotent and a unit." Glasg. Math. J., vol. 46, no. 2, pp. 227-236, 2004, doi: 10.1017/S0017089504001727.

[15] Z. Ying and J. Chen, "On quasipolar rings.” Algebra Colloq., vol. 19, no. 4, pp. 683-692, 2012, doi: $10.1142 / \mathrm{S} 1005386712000557$.

[16] Y. Zhou, "Generalizations of perfect, semiperfect and semiregular rings." Algebra Colloq., vol. 7, no. 3, pp. 305-318, 2000, doi: 10.1007/s10011-000-0305-9.

Authors' addresses

T. P. Calci

Ankara University, Department of Mathematics, 06100 Ankara, TURKEY

E-mail address: tcalcieankara.edu.tr

\section{S. Halicioglu}

Ankara University, Department of Mathematics, 06100 Ankara, TURKEY

E-mail address: halicieankara.edu.tr

\section{A. Harmanci}

Hacettepe University, Department of Mathematics, 06800 Ankara, TURKEY

E-mail address: harmanci@hacettepe.edu.tr 